\title{
Monitoring the rapid nanocrystal transformation via trapped intermediates of silica encapsulation
}

\author{
Ruoxu Wang ${ }^{1}$, Qian Wang ${ }^{1}$, Huiying Guo ${ }^{1}$ and Hongyu Chen ${ }^{1,2,3^{*}}$
}

\begin{abstract}
In transmission electron microscopy (TEM), encapsulation is an important tool to preserve the nanoparticles from aggregation, weathering, and destructive highenergy electron beam. Traditionally, encapsulation can only be used to trap end products. This study pushes the limit of in situ encapsulation so that intermediates of short time scale can be trapped. Nanocrystals of water-soluble salts were generated from a solute-induced phase separation (SIPS) process. By performing a modified Stöber encapsulation on different time points of the process, a series of intermediates can be trapped by silica shells. By arranging and comparing the intermediates, it is possible to reconstruct the growing process of those water-soluble salt nanocrystals. Moreover, an example of the transition from nanocrystal to liquid droplet was discovered, unveiling a potentially alternative route of the SIPS process. The reported technique could capture more snapshots for TEM imaging, providing crucial information on the study of nanoscale growth mechanism.
\end{abstract}

Keywords: encapsulation, hollow nanostructures, intermediate, salt nanocrystal, nanoscale droplet, kosmotrope

\section{INTRODUCTION}

The essential characteristics of nanoparticles distinct from bulk materials are their shape, size, and structural uniformity [1-3], which largely determine the properties of nanoparticles such as the catalytic [4], photonic [5,6], and electric properties $[7,8]$. Among the techniques of materials characterization, electromicroscopy is the most direct and effective method for obtaining morphological information.

Given that transmission electron microscopy (TEM) requires high vacuum, there is always a concern that the dried sample may not reflect the true solution state of the nanoparticles. In particular, typical drying would lead to an increase of concentration by over a hundred fold, greatly promoting aggregation, growth, ripening, and ligand exchange [9-11]. It would remove the co-solvents in the crystal $[12,13]$, causing weathering and sometimes collapse of crystal structures. Moreover, the high-energy electron beam could also cause damage and even complete degradation of samples [14,15]. Such electron beaminduced degradation may also affect reactions in liquid cells, causing interference even in a pricy in-situ TEM experiment.
To reduce the ambiguity brought by sample preparation, a typical method is to greatly dilute the solution sample before drying, so as to reduce the probability of unintended growth and aggregation [16,17]. The other approach is via encapsulation of the solution species, so that the resulting shell would retain the original shape and help distinguishing the subsequent processes of drying $[8,18,19]$.

Encapsulation as a technique of trapping intermediates has been applied to study the solution state of nanoparticles [20,21], nanodroplets [22,23], and surfactant micelles [24,25]. In comparison to surface ligands or surfactants, an encapsulating shell is obviously more robust against growth, etching, and aggregation. Even when the interior is completely etched, the remaining shell would still preserve the original shape and boundary conditions [26,27]. Given the slow processes of polymer and silica encapsulation, typically the solution species are quenched from the preparative solution, to allow the preservation by slow encapsulation $[21,23,28]$.

In our recent work, we discover that silica encapsulation could preserve the transient intermediates of coalescing liquid nanoparticles, in the process of solute-induced phase separation (SIPS) [22]. In this work, we aim to push the limit of in situ encapsulation, to trap intermediates of the shortest time scale. Silica encapsulation is confirmed as a rapid means of preserving nanocrystals and nanodroplets. More specifically, intermediates of $\left(\mathrm{NH}_{4}\right)_{2} \mathrm{SO}_{4}$ nanocrystals with changing morphology are observed in the early stages of SIPS. This capability allows us to study the transformation from salt nanocrystals to nanoscale droplets of salt solutions, revealing fundamental steps lying behind the phase separation process.

\section{RESULTS AND DISCUSSION}

Through microencapsulation, we captured a series of ammonium sulfate nanocrystals evolving into the heart-shape from a water-ethanol mixture. Specifically, excessive ammonia solution was added into an ethanol solution of sulfuric acid thickened by polyvinylpyrrolidone (PVP). The mixture was incubated for $1 \mathrm{~h}$ for the $\left(\mathrm{NH}_{4}\right)_{2} \mathrm{SO}_{4}$ to thoroughly precipitate and grow, and then tetraethyl orthosilicate (TEOS) was added to initiate the silica encapsulation. After $6 \mathrm{~h}$, the product was centrifuged and washed with water to remove the $\left(\mathrm{NH}_{4}\right)_{2} \mathrm{SO}_{4}$, leaving only the solidified silica nanoshells. As shown in the TEM image (Fig. 1a), heart-shape silica nanoshells (HSNs) with uniformed

\footnotetext{
${ }^{1}$ Institute of Advanced Synthesis (IAS), School of Chemistry and Molecular Engineering, Jiangsu National Synergetic Innovation Centre for Advanced Materials, Nanjing Tech University, Nanjing 211816, China

${ }^{2}$ School of Science, Westlake University, Hangzhou 310024, China

${ }^{3}$ Institute of Natural Sciences, Westlake Institute for Advanced Study, Hangzhou 310024, China

* Corresponding author (email: chenhongyu@westlake.edu.cn)
} 

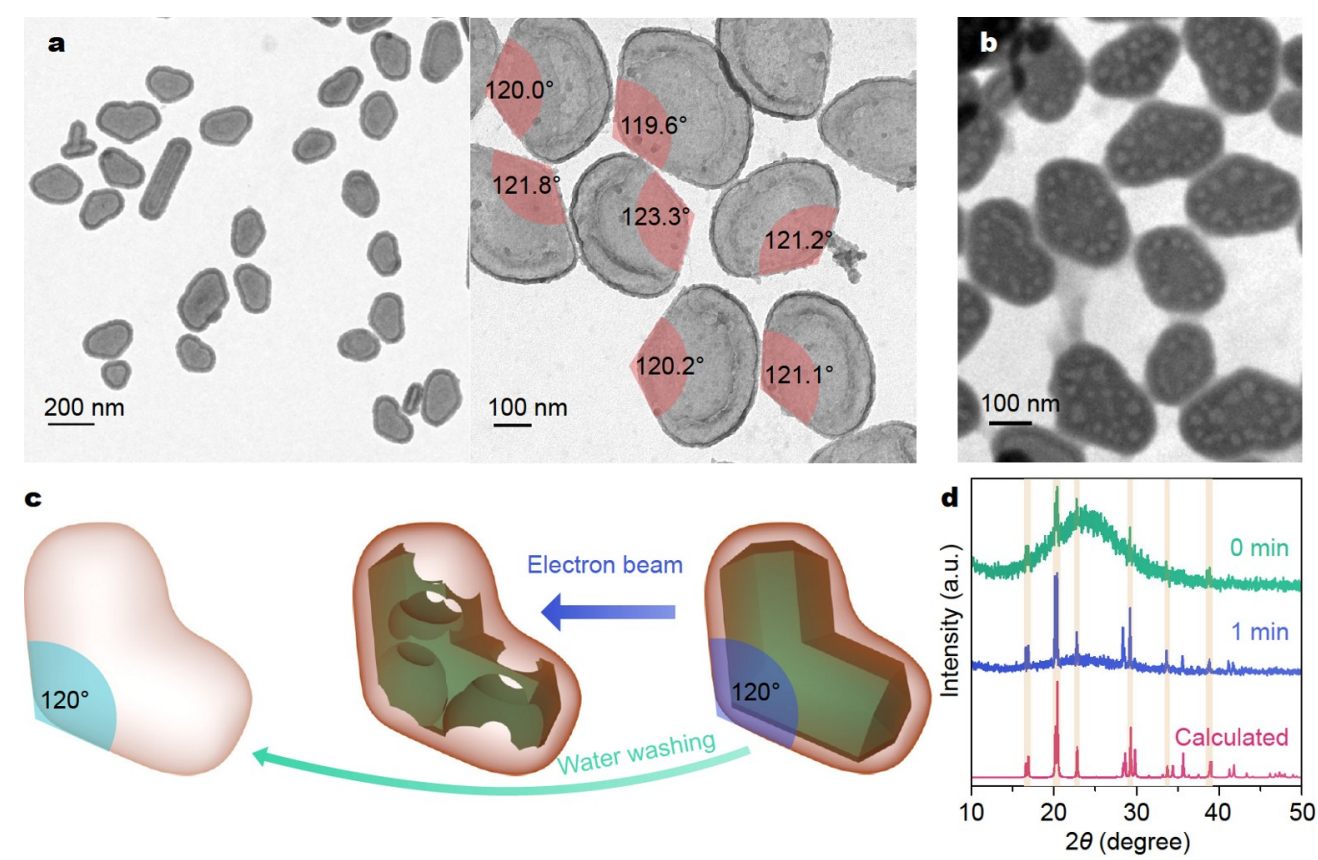

Figure 1 Trapped HSNs from $\left(\mathrm{NH}_{4}\right)_{2} \mathrm{SO}_{4}$ nanocrystals synthesized from an SIPS process. (a) TEM image, all the vertex angles are around $120^{\circ}$. (b) Bubbling effect when $\left(\mathrm{NH}_{4}\right)_{2} \mathrm{SO}_{4}$ inside the nanoshell is not removed. (c) Proposed relationship between $\mathrm{HSNs}$, bubbling nanoshells and $\left(\mathrm{NH}_{4}\right)_{2} \mathrm{SO}_{4} \mathrm{nanocrystals}$. (d) Powder XRD patterns of intermediates without removing the $\left(\mathrm{NH}_{4}\right)_{2} \mathrm{SO}_{4}$ core at different time points and the standard calculated from $\left(\mathrm{NH}_{4}\right)_{2} \mathrm{SO}_{4}$ crystal data.

sizes can be observed. A distinct darker border can be seen on each HSN, where the internal area is of lower contrast ratio. On some HSNs, a wrinkle can be observed, indicating the collapse of the hollowed nanoshell after the salt core is removed. Each HSN is of a flattened concave-clamshell shape, with the bottom edge tilting inwards, loosely resembling a V shape. Their width is around $300 \mathrm{~nm}$. The vertex angles of the nanoshells are almost exactly $120^{\circ}$, which is not likely coming from random bending and deforming of the "templates" once inside. This regularity suggests that those templates are likely to be $\left(\mathrm{NH}_{4}\right)_{2} \mathrm{SO}_{4}$ nanocrystals.

In order to retain the $\left(\mathrm{NH}_{4}\right)_{2} \mathrm{SO}_{4}$, the product was washed by dichloromethane instead of water. Under TEM, the iconic heartshape with a $120^{\circ}$ vertex angle can still be observed (Fig. $1 \mathrm{~b}$ and Fig. S1), indicating the validity of silica encapsulation on shape preservation. Additionally, low contrast "bubble" areas can be observed forming in real time. This bubbling effect can be slowed down if the strength of electron beam was significantly reduced, suggesting that it is an electron beam-induced degradation.

In a control experiment, the encapsulation step was omitted, and the resulting "naked" $\left(\mathrm{NH}_{4}\right)_{2} \mathrm{SO}_{4}$ nanocrystals were observed under TEM (Fig. S2a). Only blurry clamshell outlines can be discerned with very low electron beam strength and prolonged exposure time, otherwise the sample would instantly bubble into an unrecognizable state. Larger $\left(\mathrm{NH}_{4}\right)_{2} \mathrm{SO}_{4}$ crystals at micron size were also observed under TEM (Fig. S2b). This time we were able to see how electron beam turned the sample from sharp cornered polygons into an indiscernible collection of bubbles. These bubbling phenomena correspond to what happened before in the silica nanoshells (Fig. 1b, c). Ammonium sulfate is known to be thermally unstable, and such instability has been amplified at nanoscale. Hence, its nanocrystals cannot withstand the bombardment of electrons, which renders characterizations such as high-resolution TEM and electron diffraction impossible. It marks the significance of encapsulation in studying the morphology of such unstable nanoparticles.

On the other hand, the synthesis of water-soluble salt nanocrystals has long been a challenge $[29,30]$. Even the nanocrystals of relatively more stable salts would suffer from low contrast and blurry borderlines under TEM. The encapsulation technique in this work could synthesize such salt nanocrystals with ease and preserve their shapes at the same time, enhancing the window of observation. It also serves as a potential platform for synthesizing and studying water soluble nanocrystals.

Moreover, the time scale of silica encapsulation has been demonstrated by capturing intermediates of growing $\left(\mathrm{NH}_{4}\right)_{2} \mathrm{SO}_{4}$ nanocrystals. In the aforementioned experiment, encapsulation was conducted $1 \mathrm{~h}$ later, after the reaction settled down. Hence only the final product was captured, similar to those in the traditional methods. It only serves as a standard sample, marking the final stage of the growing process. By applying encapsulation on different time points, growing intermediates can be trapped. Specifically, TEOS was added right before the addition of $\mathrm{NH}_{3}$ (set as "0 min after the addition"), and 1 min after the addition of $\mathrm{NH}_{3}$, respectively. The standard sample is viewed as $60 \mathrm{~min}$ after the addition, accordingly. After removing the salt core, the 0 -min product shows hollow nanocubes under TEM (Fig. 2e). Their sizes range from 50 to $100 \mathrm{~nm}$. The 1 -min product (Fig. 2f) shows a collection of HSNs, rods and a few Vshaped particles. The HSNs are similar with those from the standard sample, with the $120^{\circ}$ vertex angle. The rods have curved tips. Their width is around $50-70 \mathrm{~nm}$, with length ranging from 100 to $400 \mathrm{~nm}$. There is no constant aspect ratio among the rods. The legs of $\mathrm{V}$-shaped particles have similar width with the rods. The angle of the corners is either $120^{\circ}$ or 


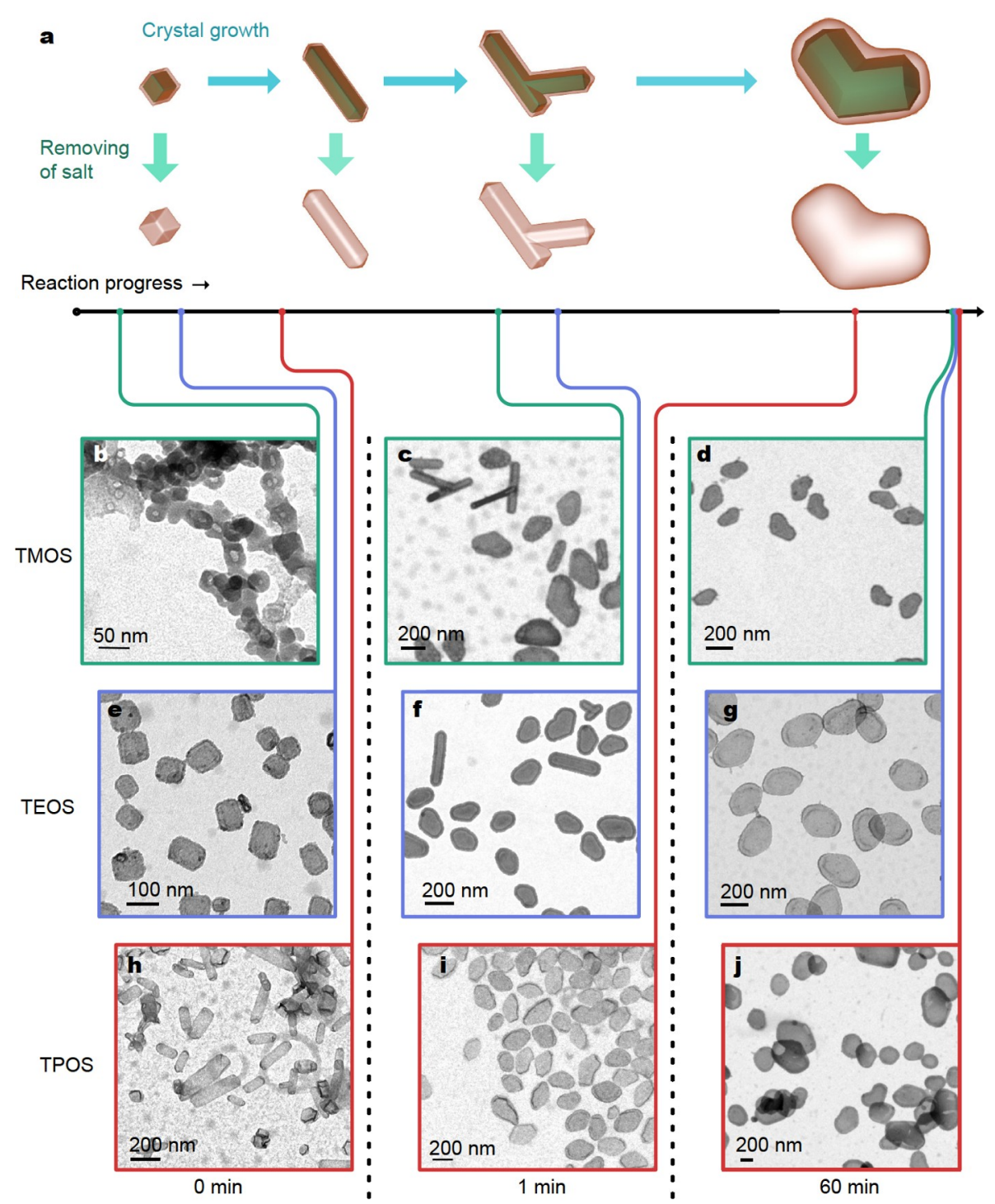

Figure 2 Shape evolution of $\left(\mathrm{NH}_{4}\right)_{2} \mathrm{SO}_{4}$ nanocrystals. (a) Schematics of the salt nanocrystals evolving from cube to rod, to V-shaped particle, and finally into HSNs. Removal of salt leads to corresponding silica nanoshells. TEM images of the intermediates trapped by TMOS at (b) 0 min, (c) 1 min, and (d) 60 min; intermediates trapped by TEOS at (e) $0 \mathrm{~min}$, (f) $1 \mathrm{~min}$, (g) $60 \mathrm{~min}$; and intermediates trapped by TPOS at (h) $0 \mathrm{~min}$, (i) $1 \mathrm{~min}$, (j) $60 \mathrm{~min}$. All intermediates have been arranged on the timeline.

$60^{\circ}$ (Fig. S3). T-shaped particles can also be observed. They are expected to be further grown $\mathrm{V}$-shaped particles, since the angles between the intersections are also $120^{\circ}$ and $60^{\circ}$, which are a pair of supplementary angles.

More intermediates have been trapped for additional information, more specifically, by switching TEOS with other siloxanes in the microencapsulation process. Siloxanes such as tetramethyl orthosilicate (TMOS) and tetrapropyl orthosilicate (TPOS) have different hydrolyzation rates, resulting in different trapping speeds. Hence, TMOS captures earlier intermediates than TEOS does if added at the same time point, thanks to its faster hydrolyzation. Similarly, TPOS captures later intermediates than TEOS does.

As shown in the TEM images, at $0 \mathrm{~min}$, TMOS traps much smaller products (Fig. 2b). The inner diameters of the silica hollow nanostructures are around $10-20 \mathrm{~nm}$, and the smallest can reach below $7 \mathrm{~nm}$. It indicates the small size of the intermediates within, as well as the great trapping capability of the encapsulation technique. On the other hand, TPOS traps round tipped nanorods (Fig. 2h), with lengths of 150-300 nm and widths of $40-60 \mathrm{~nm}$, which are similar to rods in the TEOS $1 \mathrm{~min}$ experiments. At $1 \mathrm{~min}$, TMOS gives a combination of rods, HSNs, and V-shaped particles, all of which are similar to those in the TEOS experiments. The only difference is that the proportion of rods is higher. As for TPOS, when encapsulated at $1 \mathrm{~min}$ (Fig. 2i), it produces only HSNs, with more smooth shapes. After $60 \mathrm{~min}$, both TMOS and TPOS capture only HSNs, similar to what TEOS does (Fig. 2d, g, j).

With the above analysis, it is possible to arrange the 9 snapshots provided by the three siloxanes, from the first to the last. As shown in Fig. 2a, TMOS 0 min captures the first snapshot, followed by TEOS $0 \mathrm{~min}$, then TPOS $0 \mathrm{~min}$, followed by TMOS $1 \mathrm{~min}$, TEOS $1 \mathrm{~min}$, and TPOS $1 \mathrm{~min}$, respectively. All the three 60 min snapshots are considered as the same since the prolonged standing overrides the difference between siloxanes. From those snapshots, it is possible to hypothesize the shape evolution of 
$\left(\mathrm{NH}_{4}\right)_{2} \mathrm{SO}_{4}$ nanocrystals: from small cubes to rods, then developing into $\mathrm{V}$-shaped structures, and finally growing into HSNs.

Since ammonium sulfate cannot withstand electron beam, powder X-ray diffraction (XRD) data were collected from the typical samples, where the encapsulation was conducted at 0 and $1 \mathrm{~min}$ by TEOS without removing the $\left(\mathrm{NH}_{4}\right)_{2} \mathrm{SO}_{4}$ core, respectively. As is shown in Fig. 1d, though masked by a broad peak, the 0 min sample still shows several discernible peaks consistent with those from the $1 \mathrm{~min}$ sample, which is correspondent to the calculated pattern from $\left(\mathrm{NH}_{4}\right)_{2} \mathrm{SO}_{4}$ crystals [31]. It suggests that crystalline salts have been captured and preserved by the silica encapsulation process in both intermediates.

It is traditionally believed that the Stöber reaction requires a relatively long time. In the literature, such reactions usually take several hours to finish. However, the above experiments suggest otherwise. Since the growing salt crystals are an acid-base neutralization reaction, which is usually considered to be fast. The fact that the intermediates have been captured indicating that the Stöber reaction could rapidly lock the encapsulated objects. Hence, the time scale of the Stöber reaction is evaluated.

It is to be mentioned that Stöber reactions can also be initiated by alkali metal hydroxides, such as $\mathrm{NaOH}$ and $\mathrm{KOH}$. Therefore, silica encapsulation can also be applied to ammonium-sensitive systems to prevent the interference from undesirable coordinations. However, Stöber silica encapsulation requires a basic condition to occur. Hence, trapping base-sensitive products is yet a challenge.

In an experiment similar to the TEOS 0 min one, 1 min after the addition of TEOS, the solution was centrifuged at $20,000 \times \mathrm{g}$ for $1 \mathrm{~min}$, and the precipitant was washed by $0.1 \mathrm{~mol} \mathrm{~L}^{-1} \mathrm{HCl}$. From the TEM image, the sample shows residue which is insoluble to water (Fig. S4). The precipitant was dissolved by concentrated $\mathrm{NH}_{3}$ and analyzed by inductively coupled plasma mass spectrometry, and silicon element has been detected. It shows that $9.69 \%$ silicon element has transferred from TEOS into insoluble products. It suggests that silica in a Stöber reaction could form in less than $2 \mathrm{~min}$; even though silica at such early stage may not be strong enough to retain any specific shapes, it may be enough to block the surface of the encapsulated object. It was hypothesized that partially hydrolyzed siloxanes could show amphiphilicities similar to surfactants [32]. These silica oligomers could gather on the surface of particles in the solution. We believe that these oligomers could quickly crosslink into a layer of ultrathin silica. It will block the particle surface from further growth, trapping the intermediates.

When the above method was applied to an SIPS process triggered by triammonium citrate $\left(\left(\mathrm{NH}_{4}\right)_{3} \mathrm{Ct}\right)$, a series of intermediates were trapped, representing a transition from nanocrystal to droplets (Fig. 3a). The SIPS refers to the phase separation from a monophase homogeneous liquid mixture when a kosmotrope is introduced. Such mixtures in general consist of water and a water-miscible organic solvent. The kosmotrope in this context usually means water-soluble carbohydrates and salts of which the anion has high charge density $[22,33,34]$. More specifically, in the experiment, excessive $\mathrm{NH}_{3}$ was introduced into an isopropanol solution of citric acid which is thickened by hydroxypropyl cellulose (HPC). Then TEOS was introduced to initialize the encapsulation at different time points to capture corresponding intermediates.

All TEM images were taken after washing with water, so all the cores have been removed, leaving only the silica shells pre- serving their shape. When TEOS was introduced at $0 \mathrm{~min}$, the image of the product shows polygonal hollow structures with sharp corners ranging from 30 to $100 \mathrm{~nm}$ (Fig. 3b). The XRD pattern of the unwashed sample corresponds to that of standard $\left(\mathrm{NH}_{4}\right)_{3} \mathrm{Ct}$ crystals (Fig. S5). Both evidences indicate that salt nanocrystals have been trapped. For the intermediate trapped at $1 \mathrm{~min}$, its TEM image shows some interesting results (Fig. 3c). Along with polygonal silica shells similar to those from the previous experiment, some broken hollow spheres can also be observed. They are of significant larger sizes, with diameters larger than $200 \mathrm{~nm}$. The broken spheres represent the scenario of osmosis pressure damage in hypotonic cells, where a hollow capsule was bloated by the expanding liquid within. This similarity may suggest that osmosis pressure can affect the structure at nanoscales. It shows great potential as a tool in manipulating nanostructures in the future. In the experiment that encapsulation was conducted after $30 \mathrm{~min}$ (Fig. 3d), only large, whole hollow spheres can be observed in the TEM image of the products. Their diameters are around $300-400 \mathrm{~nm}$, considerably larger than products in previous experiments. From the first to the last, trapped intermediates show an obvious increase in size, and more importantly, a transition from nanocrystal to droplets.

We hypothesize that in such an SIPS system (Fig. 3a), when ammonia reacts with citric acid in a water-isopropanol mixture, the resulting salt firstly nucleates as crystals. Then water starts to condense on the surface of the salt nanocrystals through heterogeneous nucleation. The process may be similar to raindrop nucleation since heterogeneous nucleation usually has a considerably lower energy barrier than homogeneous nucleation does. The water starts to dissolve the salt core. When the entire salt crystal surface is covered by an extremely thin layer of water, the nucleus becomes a droplet and starts to extract water from the water-isopropanol mixture. This will cause an expansion of the water domain, which bloats the silica shell, resulting in broken sphere intermediates. Hence, the whole process can be separated into three stages: (1) the emerging of salt crystals; (2) the condensation of water on salt into droplets, and (3) the growth of droplet through extraction of water. Thanks to the rapid trapping through silica encapsulation, the transition process can be recovered. Otherwise, such transient kinetic moment can be buried under the thermodynamic narratives.

In our hypothesis, we assume each of the three stages happens separately. One only occurs after the previous one has finished. Hence, we would focus on stage 2 which is the key step in the transition from solid crystals to droplets. It is described as a heterogeneous nucleation of water on the surface of a solid core $[35,36]$, right before water starts to dissolve the salt. If this key step occurs faster than the encapsulation, it would be impossible to trap the crystalline intermediate. Therefore, analysis of the heterogeneous nucleation rate would be crucial, which can be described by Equation (1):

$\frac{\mathrm{d} N}{\mathrm{~d} t}=A \exp \left(-\frac{\Delta G^{*}{ }^{*}{ }^{2}{ }^{2}}{k_{\mathrm{B}} T}\right)$,

where $\Delta G^{*}{ }_{\text {hetero }}$ refers to the critical free energy of heterogeneous nucleation of water on salt; $k_{\mathrm{B}}$ is the Boltzmann's constant, $T$ is the temperature. $A$ is a pre-exponential factor which is considered to be constant at a given condition. The temperature is also kept the same. Hence, the key to the nucleation rate is

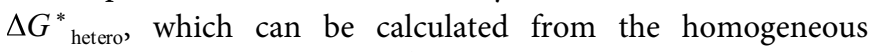
nucleation critical energy $\Delta G^{*}$ by Equation (2) [35]: 


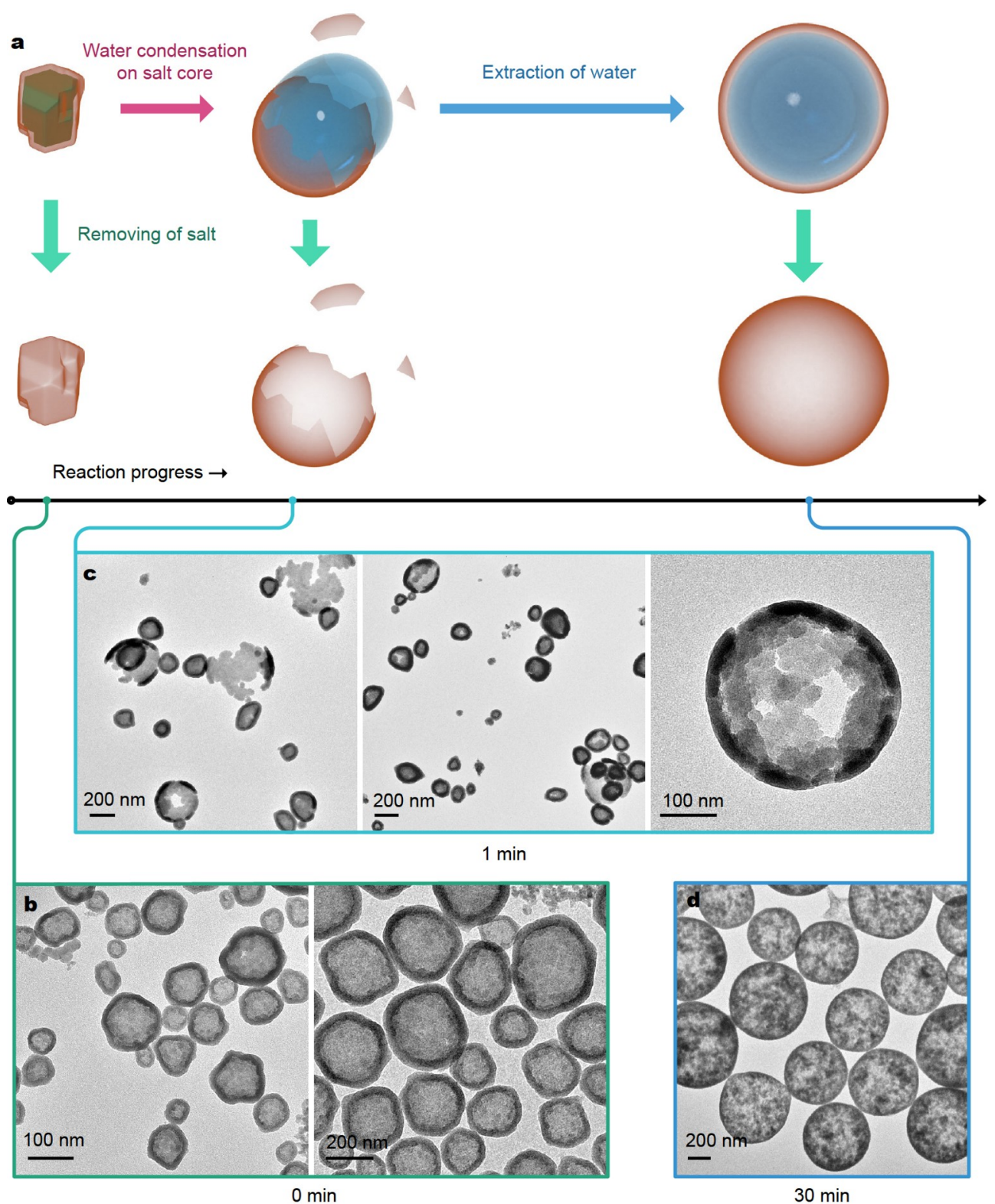

Figure 3 The transition from $\left(\mathrm{NH}_{4}\right)_{3} \mathrm{Ct}$ nanocrystals to salty liquid droplets captured by silica nanoshells. (a) Schematic of salt nanocrystals extracting water into droplets, and switching to droplet growth. If encapsulation is conducted at early stage, nanocrystal can be trapped. Then water condenses on the salt core and dissolves it into a salty droplet. The expanding droplet, if trapped by encapsulation, leads to broken spheres as intermediates. At the final stage, when droplet growth is completed, encapsulation traps whole hollow nanospheres. TEM images of intermediates trapped at (b) 0 min as nanocrystals, (c) 1 min as broken spheres, and (d) 30 min as whole hollow nanospheres. All intermediates have been arranged on the timeline.

$\Delta G^{*}{ }_{\text {hetero }}=\frac{1}{4}(2+\cos \theta)(1-\cos \theta)^{2} \Delta G^{*}$,

where $\theta$ is the contact angle of salty water on the salt. Since the nucleation happens in a ternary system, the contact angle between two phases follows Young's relation:

$\cos \theta=\frac{\gamma_{\mathrm{as}}-\gamma_{\mathrm{wa}}}{\gamma_{\mathrm{ws}}}$

where $\gamma_{\mathrm{as}}, \gamma_{\mathrm{wa}}$ and $\gamma_{\mathrm{ws}}$ refer to the surface energies between alcohol phase and salt, aqueous and alcohol phase, aqueous phase and salt, respectively (Fig. 4a). Here, we assume the condition change of alcohol phase due to condensation is ignorable. $\gamma_{\text {wa }}$ can be considered zero for alcohols which are infinitely miscible, such as ethanol and isopropanol. Therefore, Equations (1-3) can be combined into

$$
\frac{\mathrm{d} N}{\mathrm{~d} t}=A \exp \left(-\left(2+\frac{\gamma_{\mathrm{as}}}{\gamma_{\mathrm{ws}}}\right)\left(1-\frac{\gamma_{\mathrm{as}}}{\gamma_{\mathrm{ws}}}\right)^{2} \frac{\Delta G^{*}}{4 k_{\mathrm{B}} T} .\right.
$$

Through Equation (4) it can be explained why thickening is crucial in trapping nanocrystal intermediates. It increases $\gamma_{\text {as }}$ which leads to decreased water nucleation speed, making the trapping of crystal stage possible.

Further control experiments correspond to the trend of 
a
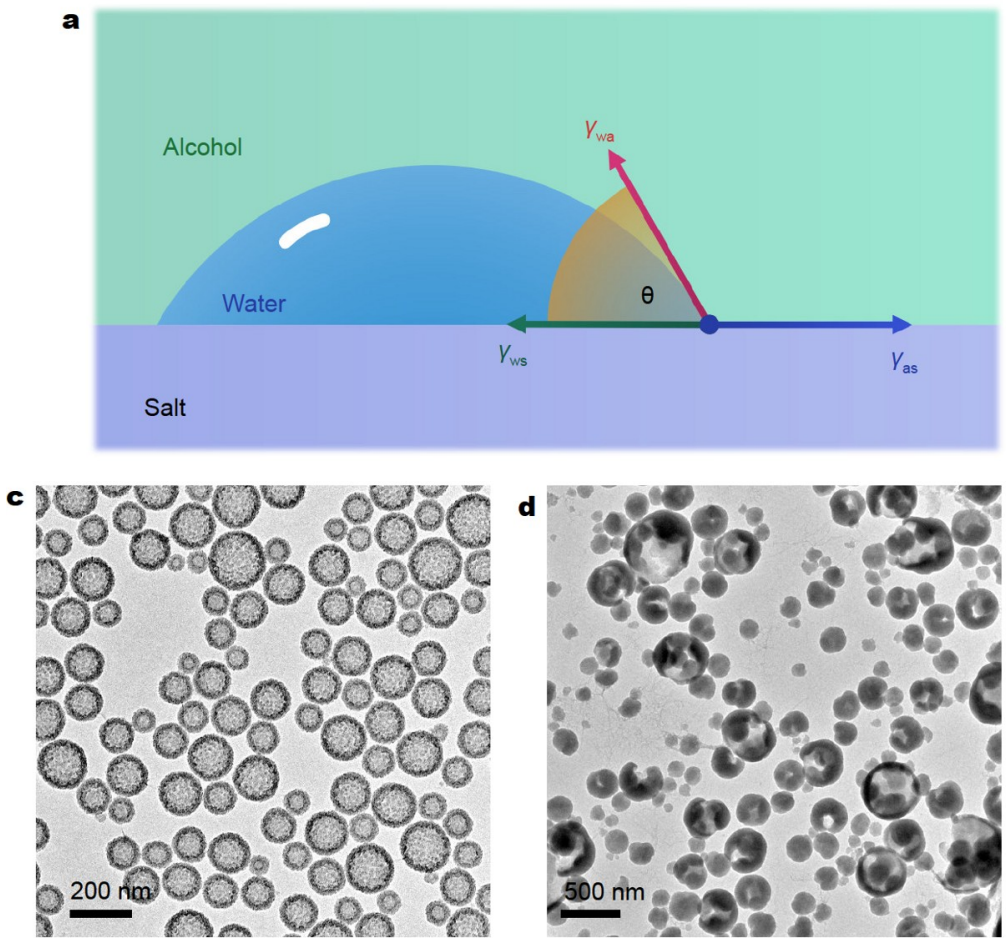
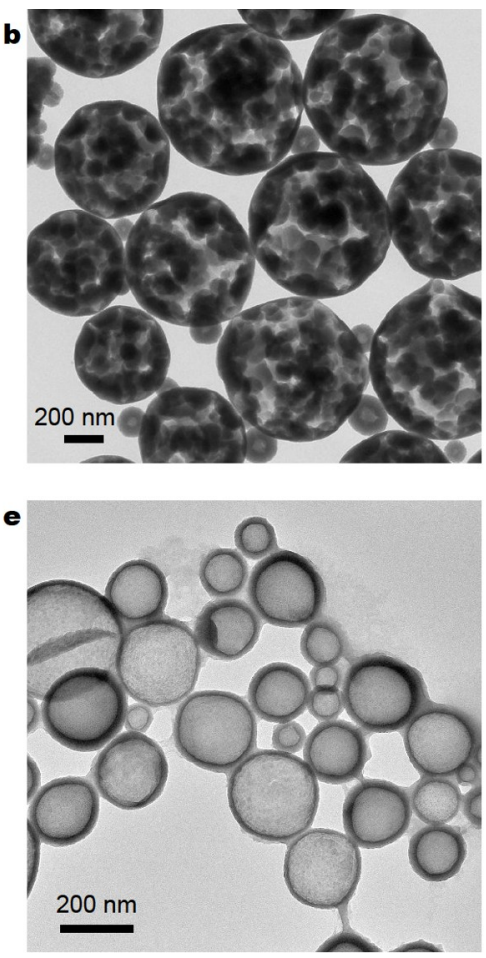

Figure 4 Explanation of Equation (4) and TEM images of further control experiments. (a) Schematic representing Young's relation in a salt-water-alcohol ternary phase system. (b) TEM image of intermediates trapped without thickeners. (c) TEM image of intermediates trapped in a water-ethanol SIPS system. (d) TEM image of intermediates trapped in a modified water-ethanol SIPS system when thickening agent was increased to 10 times where broken spheres can be observed. (e) TEM image of intermediate trapped in a water- $n$-pentanol SIPS system.

Equation (4). When the amount of HPC was decreased to $1 / 100$, no crystalline intermediates can be trapped (Fig. 4b). Encapsulation only yield in hollow nanospheres. This is due to the increased water heterogeneous nucleation rate surpassed the time resolution of the Stöber encapsulation.

We also applied Equation (4) to explain a seemingly discrepancy between the water-isopropanol system and the waterethanol system reported previously [22]. In the water-ethanol system, with the same condition, only hollow nanosphere can be trapped (Fig. 4c). However, when HPC concentration was increased to 10 times, broken spheres can be trapped (Fig. $4 \mathrm{~d}$ ), which is similar to the product in the $1 \mathrm{~min}$ experiment in the water-isopropanol system. Since broken spheres are considered as the intermediate when salty droplet is extracting water, it can be implied that stage 3 may be present in the water-ethanol system.

Another control experiment has been tested in a water- $n$ pentanol system. These two solvents are partly miscible, where $n$-pentanol can accommodate around $7 \%$ water per volume. In the experiment, only nanospheres are trapped (Fig. 4e). There are two possible explanations. On one hand, since pentanol is only partially miscible with water, $\gamma_{\text {wa }}$ cannot be ignored. It leads to a decrease in $\cos \theta$ and exponential increase in the water nucleation rate, surpassing the time resolution of encapsulation. On the other hand, the process of pentanol dissolving water takes some time. Therefore, water may first be dispersed in the pentanol "oil" like an emulsion, then gradually dissolves by pentanol, leading to a one-phase mixture. In this process, droplet forms without any kosmotrope. The already existed water droplets could directly extract the salt, instead of being extracted.
Therefore, no crystal stage would take place. An experimental fact indicates the latter might be closer to the truth, since when pure water is added to pentanol, a cloudy state can be observed, which indicates the presence of water droplets in pentanol even without $\left(\mathrm{NH}_{4}\right)_{3} \mathrm{Ct}$.

A comparison between our result and facts in the literature suggests that there may be multiple routes in an SIPS process, even if the final products are all hollow spheres. The absence of a trapped crystal intermediate may be due to either the insufficient time resolution of the encapsulation, or the absence of such crystals at the first place. Our hypothesis is that when the kosmotrope has high water affinity, such as carbohydrates, there might not be a crystal stage, and the kosmotrope will be "pushed" out along with water; if the kosmotrope is "hated" too much by the water-miscible organic solvent, it may crystalize first, followed by the condensation and extraction of water; if the organic solvent is only partially miscible with water, water droplet would appear first, then extract the kosmotrope, like in a traditional emulsion system.

\section{CONCLUSIONS}

In summary, this study proved that encapsulation can be applied to trap intermediates of growing salt nanocrystals and droplets rather than mere end products. The time scale of Stöber encapsulation has been validated to be able to capture early-stage intermediates. Also, the evolving of $\left(\mathrm{NH}_{4}\right)_{2} \mathrm{SO}_{4}$ nanocrystals from cubes to HSNs, as well as the transition from nanocrystals to droplets of ammonium citrate have been demonstrated by the encapsulation technique and the intermediates it trapped. A potential alternative route of the SIPS process has been proposed 
from the information provided by the intermediates. We believe the encapsulation technique could be an important tool in facilitating future mechanism studies.

Received 21 November 2021; accepted 13 December 2021; published online 27 January 2022

1 Skrabalak SE, Chen J, Sun Y, et al. Gold nanocages: Synthesis, properties, and applications. Acc Chem Res, 2008, 41: 1587-1595

2 Ni B, Shi Y, Wang X. The sub-nanometer scale as a new focus in nanoscience. Adv Mater, 2018, 30: 1802031

3 Choi SH, Kim EG, Park J, et al. Large-scale synthesis of hexagonal pyramid-shaped $\mathrm{ZnO}$ nanocrystals from thermolysis of $\mathrm{Zn}$-oleate complex. J Phys Chem B, 2005, 109: 14792-14794

4 Ge J, Lei J, Zare RN. Protein-inorganic hybrid nanoflowers. Nat Nanotech, 2012, 7: 428-432

5 Lim DK, Jeon KS, Hwang JH, et al. Highly uniform and reproducible surface-enhanced Raman scattering from DNA-tailorable nanoparticles with 1-nm interior gap. Nat Nanotech, 2011, 6: 452-460

6 Wang Y, Han Y, Tan X, et al. Cyclodextrin capped gold nanoparticles (AuNP@CDs): From synthesis to applications. J Mater Chem B, 2021, 9: $2584-2593$

7 Faber MS, Jin S. Earth-abundant inorganic electrocatalysts and their nanostructures for energy conversion applications. Energy Environ Sci, 2014, 7: 3519-3542

8 Sun $\mathrm{H}$, Shen $\mathrm{X}$, Yao L, et al. Measuring the unusually slow ionic diffusion in polyaniline via study of yolk-shell nanostructures. J Am Chem Soc, 2012, 134: 11243-11250

9 Standridge SD, Schatz GC, Hupp JT. Toward plasmonic solar cells: Protection of silver nanoparticles via atomic layer deposition of $\mathrm{TiO}_{2}$. Langmuir, 2009, 25: 2596-2600

10 Shrestha S, Wang B, Dutta P. Nanoparticle processing: Understanding and controlling aggregation. Adv Colloid Interface Sci, 2020, 279: 102162

11 Baraldi G, Carrada M, Toudert J, et al. Preventing the degradation of $\mathrm{Ag}$ nanoparticles using an ultrathin a- $\mathrm{Al}_{2} \mathrm{O}_{3}$ layer as protective barrier. J Phys Chem C, 2013, 117: 9431-9439

12 Peng Y, Gardner DJ, Han Y, et al. Influence of drying method on the material properties of nanocellulose I: Thermostability and crystallinity. Cellulose, 2013, 20: 2379-2392

13 Wang S, Lai Z, Tran TH, et al. Solvent exchange as a synthetic handle for controlling molecular crystals. Curr Alzheimer Resbon, 2020, 160: 188-195

14 Xin W, De Rosa IM, Ye S, et al. Effects of electron beam irradiation and hydroxyl ion concentration on morphological stability of polyethylenimine-capped gold nanoparticles. Mater Res Express, 2019, 6: 125031

15 Reyes-Gasga J, Garcia-Garcia R. Analysis of the electron-beam radiation damage of TEM samples in the acceleration energy range from 0.1 to $2 \mathrm{MeV}$ using the standard theory for fast electrons. Radiat Phys Chem, 2002, 64: 359-367

16 Wang $\mathrm{H}, \mathrm{Xu}$ J, Wang J, et al. Probing the kinetics of short-distance drug release from nanocarriers to nanoacceptors. Angew Chem Int Ed, 2010, 49: $8426-8430$

17 Sheikholeslami S, Jun YW, Jain PK, et al. Coupling of optical resonances in a compositionally asymmetric plasmonic nanoparticle dimer. Nano Lett, 2010, 10: 2655-2660

18 Chen $\mathrm{T}$, Wang $\mathrm{X}$, Alizadeh $\mathrm{MH}$, et al. Monitoring transient nanoparticle interactions with liposome-confined plasmonic transducers. Microsyst Nanoeng, 2017, 3: 16086

19 Fernandez YD, Sun L, Gschneidtner T, et al. Research update: Progress in synthesis of nanoparticle dimers by self-assembly. APL Mater, 2014, 2: 010702

20 Li JF, Huang YF, Ding Y, et al. Shell-isolated nanoparticle-enhanced Raman spectroscopy. Nature, 2010, 464: 392-395

21 Wang $\mathrm{H}$, Chen L, Feng Y, et al. Exploiting core-shell synergy for nanosynthesis and mechanistic investigation. Acc Chem Res, 2013, 46: 1636-1646
22 Wang R, Han F, Chen B, et al. Liquid nanoparticles: Manipulating the nucleation and growth of nanoscale droplets. Angew Chem Int Ed, 2021, 60: 3047-3054

23 Zhang J, Coulston RJ, Jones ST, et al. One-step fabrication of supramolecular microcapsules from microfluidic droplets. Science, 2012, 335: 690-694

24 Koh K, Ohno K, Tsujii Y, et al. Precision synthesis of organic/inorganic hybrid nanocapsules with a silanol-functionalized micelle template. Angew Chem, 2003, 115: 4326-4329

25 Zhang $\mathrm{X}$, Che S. Mesoporous silica microspheres composited with SBA-15s for resonance frequency reduction in a miniature loudspeaker. Chem Res Chin Univ, 2020, 36: 760-767

26 Cheng D, Xia H, Chan HSO. Fabrication of polymeric hollow nanospheres, hollow nanocubes and hollow plates. Nanotechnology, 2006, 17: 1661-1667

27 Bai YK, Zheng RT, Gu Q, et al. One-step synthesis of hollow $\mathrm{Cr}(\mathrm{OH})_{3}$ micro/nano-hexagonal pellets and the catalytic properties of hollow $\mathrm{Cr}_{2} \mathrm{O}_{3}$ structures. J Mater Chem A, 2014, 2: 12770-12775

28 Feng $\mathrm{Y}$, Wang $\mathrm{Y}, \mathrm{He} \mathrm{J}$, et al. Achieving site-specificity in multistep colloidal synthesis. J Am Chem Soc, 2015, 137: 7624-7627

29 Chen Q, Hood ZD, Qiu J, et al. Continuous production of water-soluble nanocrystals through anti-solvent precipitation in a fluidic device. ChemNanoMat, 2019, 5: 1131-1136

30 Annen T, Epple M. A facile synthesis of dispersable $\mathrm{NaCl}$ nanocrystals. Dalton Trans, 2009, 9731

31 Hasebe K. Studies of the crystal structure of ammonium sulfate in connection with its ferroelectric phase transition. J Phys Soc Jpn, 1981, 50: $1266-1274$

32 Yi D, Zhang Q, Liu Y, et al. Synthesis of chemically asymmetric silica nanobottles and their application for cargo loading and as nanoreactors and nanomotors. Angew Chem, 2016, 128: 14953-14957

33 Assaf KI, Nau WM. The chaotropic effect as an assembly motif in chemistry. Angew Chem Int Ed, 2018, 57: 13968-13981

34 Ball P, Hallsworth JE. Water structure and chaotropicity: Their uses, abuses and biological implications. Phys Chem Chem Phys, 2015, 17: 8297-8305

35 Thanh NTK, Maclean N, Mahiddine S. Mechanisms of nucleation and growth of nanoparticles in solution. Chem Rev, 2014, 114: 7610-7630

36 Wang $\mathrm{Y}, \mathrm{He} \mathrm{J}$, Liu C, et al. Thermodynamics versus kinetics in nanosynthesis. Angew Chem Int Ed, 2014, 54: 2022-2051

Acknowledgements This work was supported by the National Natural Science Foundation of China (21703104, 21673117 and 91956109), Nanjing Tech University (39837102), and the SICAM Fellowship from Jiangsu National Synergetic Innovation Center for Advanced Materials. The authors thank Westlake center for micro/nano fabrication for the facility support and technical assistance.

Author contributions Wang $\mathrm{R}$ and Chen $\mathrm{H}$ initiated the idea. Wang $\mathrm{R}$ hypothesized the mechanism. Wang R, Wang Q and Guo H conducted the experiments. Wang $\mathrm{R}$ and Chen $\mathrm{H}$ wrote the paper. The manuscript was written through contributions of all authors. All authors have given approval to the final version of the manuscript.

Conflict of interest The authors declare that they have no conflict of interest.

Supplementary information Experimental details and supporting data are available in the online version of the paper. 


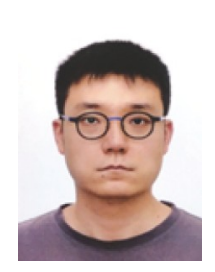

Ruoxu Wang received his BSc degree in material chemistry from Beijing Institute of Technology, MSc degree from Nanjing Tech University, and $\mathrm{PhD}$ degree from Nanyang Technological University in Singapore under the supervision of Prof. Hongyu Chen. His research interests focus on the nucleation and growth of nanoscale liquid droplets and their applications in the synthesis of hollow nanostructures, such as nanocapsules, nanoreactor, and carriers.

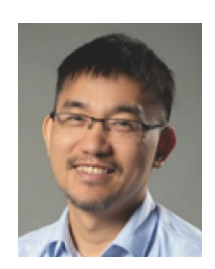

Hongyu Chen obtained his BSc degree from the University of Science and Technology of China, and then PhD degree from Yale University in 2004. He jointed Nanyang Technological University in Singapore and was promoted to a tenured associate professor. In 2016, he moved back to China and joined Nanjing Tech University, where he cofounded the Institute of Advanced Synthesis and served as executive dean. He is now a tenured full professor at Westlake University. His research interest centers on the advancement of synthetic capability at the nanoscale, more specifically on the development of synthetic methods, understanding the underlying principles, and applying these tools for novel nanostructures and new applications. See http://www.nanosynthesis.cn/ for more details.

\section{通过二氧化硅纳米包覆捕捉监测纳米晶体快速转变 过程的中间体}

王若徐 ${ }^{1}$, 王倩 ${ }^{1}$, 郭慧颓 ${ }^{1}$, 陈虹宇 $1,2,3 *$

摘要 在透射电子显微镜(TEM)表征中, 微包覆是一种重要的工具, 以 保存纳米颗粒的形貌不受聚集、风化和高能电子束的破坏. 传统上, 微 包覆只能用来捕捉最终产物. 本研究探索实时微包覆技术的极限, 以捕 捉短纳米颗粒生长过程中转瞬即逝的中间体. 本文通过溶质诱导相分 离(SIPS)体系生成了水溶性盐的纳米晶体. 利用改进的Stöber微包覆技 术捕捉了该过程中不同时间点上的一系列中间体. 并且将它们按照时 间顺序排列, 还原出该水溶性盐纳米晶体的生长过程. 除此以外, 本研 究还捕捉到了一例从盐纳米晶体到液滴的转变过程, 展示出了SIPS体 系可能存在一种与之前推测不同的变化途径. 该微包覆技术可便捷地 捕捉纳米颗粒生长中不同阶段的“快照”以便进行TEM表征，为纳米级 生长机理研究提供了更多关键信息. 